\title{
Abstract: Erweiterung des Bildgebungsbereiches bei der Magnetpartikelbildgebung durch externe axiale Verschiebungen
}

\author{
Patryk Szwargulski ${ }^{1,2}$, Nadine Gdaniec ${ }^{1,2}$, Matthias Graeser ${ }^{1,2}$, \\ Martin Möddel $^{1,2}$, Florian Griese ${ }^{1,2}$, Tobias Knopp ${ }^{1,2}$ \\ ${ }^{1}$ Abteilung für Biomedizinische Bildgebung, Universitätsklinikum \\ Hamburg-Eppendorf \\ ${ }^{2}$ Institut für Biomedizinische Bildgebung, Technische Universität Hamburg \\ p.szwargulski@uke.de
}

Die Magnetpartikelbildgebung (engl. Magnetic-Particle-Imaging, MPI) ist eine Bildgebungsmodalität, die auf der Darstellung super-paramagnetischer Nanopartikeln unter Verwendung von statischen und dynamischen Magnetfeldern basiert [1]. Das Bildgebungsverfahren weist eine hohe zeitliche Auflösung von über 40 Volumen pro Sekunde auf und ist mit einer Detektionsgrenze von $5 \mathrm{ng}$ Eisen [2] ein höchst sensitives Verfahren, mit dem viele medizinische Applikationen adressiert werden können. Als Einschränkung ist der, aus Sicherheitsgründen [3] auf wenige Kubikcentimeter beschränkte, Bildgebungsbereich zu nennen. Um diese Limitierung zu umgehen, können zusätzliche Felder geschaltet und/oder das Objekt mithilfe einer externen Verschiebung durch den Bildgebungsbereich bewegt werden. Im Rahmen dieser Arbeit wurde ein Rekonstruktionsverfahren entwickelt, welches sowohl bei statischen als auch bei dynamischen Objekten genutzt werden kann und die Rohdaten in der Form bearbeitet, dass der Messfeldverschub berücksichtigt wird. Mit der entwickelten Methode konnten sowohl statische 3D Phantomdaten als auch in-vivo Messdaten eines dynamischen Mausexperimentes $(3 \mathrm{D}+\mathrm{t})$ erfolgreich rekonstruiert werden [4].

\section{Literaturverzeichnis}

1. Gleich B, Weizenecker J. Tomographic imaging using the nonlinear response of magnetic particles. Nature. 2005;435(7046):1214 - 1217.

2. Graeser M, Knopp T, Szwargulski P, et al. Towards picogram detection of superparamagnetic iron-oxide particles using a gradiometric receive coil. Sci Rep. 2017;7:6872.

3. Saritas EU, Goodwill PW, Zhang GZ, et al. Magnetostimulation limits in magnetic particle imaging. IEEE Trans Med Imaging. 2013;32(9):1600 - 1610.

4. Szwargulski P, Gdaniec N, Graeser M, et al. Enlarging the field of view in magnetic particle imaging using a moving table approach. Proc SPIE. 2017; p. 10578-51. 